\title{
Control of virtual environments for young people with learning difficulties
}

\author{
Tanja Lannen, Dr. David Brown and Dr. Heather Powell \\ Dept. of computing, Nottingham Trent University, UK, Tanja.lannen@ntu.ac.uk
}

\begin{abstract}
Purpose: The objective of this research is to identify the requirements for the selection or development of usable virtual environment (VE) interface devices for young people with learning difficulties.

Method: A user-centred design methodology was employed, to produce a design specification for usable VE interface devices. Details of the users' cognitive, physical and perceptual abilities were obtained through observation and normative assessment tests.

Conclusions: A review of computer interface technology, including virtual reality and assistive devices, was conducted. As there were no devices identified that met all the requirements of the design specification, it was concluded that there is a need for the design and development of new concepts. Future research will involve concept and prototype development and user-based evaluation of the prototypes.
\end{abstract}

\section{Introduction}

Recent research in virtual environment (VE) applications for people with leaming difficulties has highlighted usability difficulties with the computer interface devices, which are used to perform the VE tasks. For example, from an evaluation of VEs, developed to teach independent living skills to people with learning difficulties, it was found that individuals differed in the amount of support required to use the input devices; joystick for navigation and mouse for interaction [1]. This paper describes research undertaken to identify the requirements for the selection or development of usable VE interface devices for young people with learning difficulties.

\section{VIRTUAL ENVIRONMENTS}

VEs have been found to be of educational benefit for people with learning difficulties. Before describing these benefits, what are VEs? They are three-dimensional computer simulations, which respond in real time to the activity of their users, see figure 1 . One of the first applications of VE technology was in flight simulation to train pilots within a safe environment. Their use is continually progressing in many areas, such as architecture, medicine, rehabilitation and education. There are two independent phases of operation within a VE: navigation and interaction. Navigation, with 2 degrees of freedom, allows movement forwards, backwards and turning to the left or right. Interaction includes activating VE objects (i.e. opening a door), moving VE objects from one place to another or using one object with another (i.e. using a spoon to take some sugar from a sugar bowl). The sense of presence within a VE is dependent on the input and display devices used. A high level of participant immersion can be achieved using a head-mounted display with a specialised input device, such as a data-glove. Desktop VE systems are also in widespread use, which utilise a computer monitor to display the VE and standard input devices, such as a joystick, mouse or keyboard, see figure 2. This hardware combination can also lead to a sense of presence and generally, desktop systems are preferred when working with people with learning difficulties due to the unresolved health and safety issues and high cost associated with head-mounted display units. 


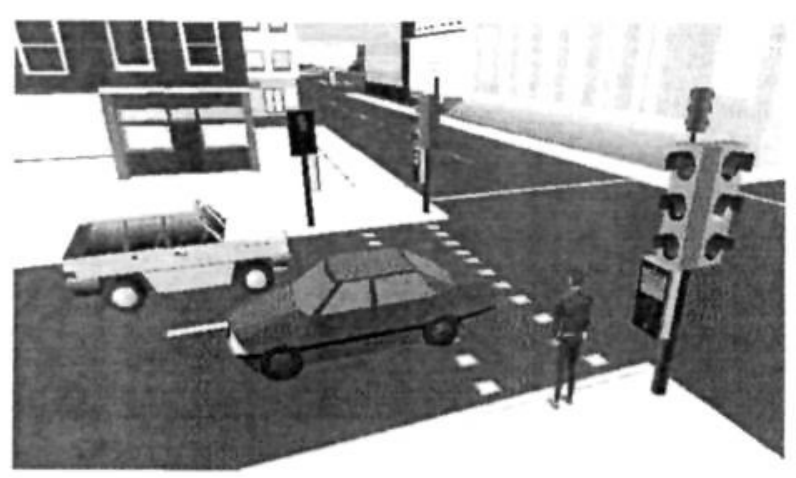

Figure 1 The virtual city

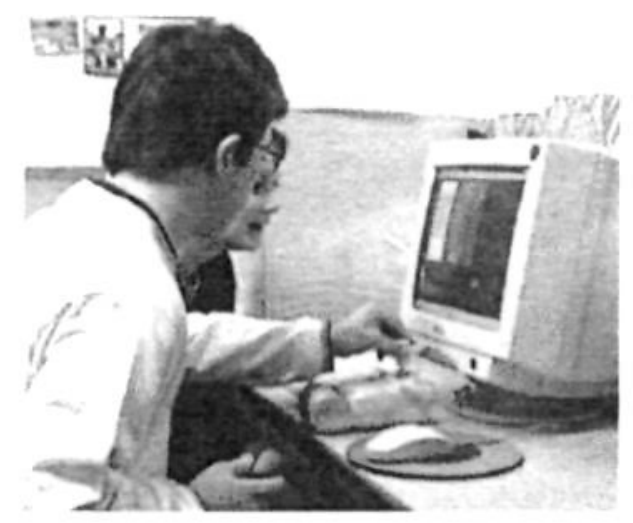

Figure 2 Desktop VE system

\section{VE BENEFITS}

Research has indicated numerous benefits in the use of VEs for the education and training of people with learning difficulties [2]. They encourage active participation in learning and give the user control over the learning process. They facilitate playful activity, by allowing individuals to learn by making mistakes without suffering the consequences of their errors. VEs are described in terms of realistic and graphical representations of the real world. Hence, they avoid abstract thought, which has been found to be particularly difficult for people with learning difficulties, who are often described as 'concrete thinkers' [3]. Finally, they can minimise the effects of many physical disabilities and allow students to take part in activities or visit places that might be inaccessible to them in real life.

\section{MOST SUITABLE INPUT DEVICES}

Studies on the most appropriate methods of VE control for people with learning difficulties have been conducted. Hall [4] concluded that a joystick, limited to two simultaneous degrees of freedom, had the greatest utility in VE navigation tasks. A study by Brown et al [5] found that the joystick was more suitable for navigation tasks than the keyboard or mouse. The touch-screen and mouse were assessed for interaction tasks and the students coped very well with both devices, however, difficulties were found in using the touch-screen to interact with small objects and with calibration of this device. From these studies it can be concluded that, from the range of input devices tested, the joystick and mouse are the most suitable navigation and interaction devices respectively. 


\section{USABILITY DIFFICULTIES}

Neale et al [6] conducted an evaluation of VEs for the education of children with severe learning difficulties. The devices utilised in this study were the joystick for navigation tasks and the mouse or touch-screen for interaction tasks. It was found that:

- Restricted movement space was difficult to navigate and led to user frustration

- Teacher assistance was required for some interaction tasks

It is important to note that participant selection for this study was based partly on ability to control the input devices. Although the navigation difficulties found were software related, it is the author's belief that, the overall usability of the system would be enhanced by refining the input devices as well as the software interface. In the aforementioned study by Cobb et al [1], it was found that individuals differed in the amount of support required to use the input devices; joystick for navigation and mouse for interaction. It was also stated that navigation was found to be one of the most difficult tasks to do. This research by Neale et al [6] and Cobb [1] has shown that there are usability difficulties with the input devices, which have been found to be the most suitable for navigation and interaction tasks, from the range of devices tested by Hall [4] and Brown et al [5].

\section{SOLUTIONS}

In the aforementioned study by Brown et al [5] the following requirements for input device design or refinement were identified: operable by people with fine-motor difficulties, modifiable, robust, easy to calibrate and affordable. Lannen [7] developed a prototype interface device, the Mojo interactive seat, which meets some of these requirements: operable by people with fine-motor difficulties, modifiable and affordable, see figure 3 . Mojo was compared with a joystick for control of a VE navigation task by students with moderate to severe learning difficulties, some of whom were also physically impaired [8]. From the results it was clear that less disorientation occurred when using Mojo, however, both devices would require refinement to provide an adequate solution to this human-VE interaction problem.

A review of computer interface technology, including virtual reality and assistive devices, was conducted. Assistive devices have been designed to improve access to computers for people with a wide range of disabilities, for example voice and gesture recognition, eye and head tracking and brain wave control. However, no research has been found which investigates the use of such devices for the control of VEs for people with learning difficulties. It has also been expressed that the cost of the technology mentioned would currently be too great for most individuals and some organisations [5].
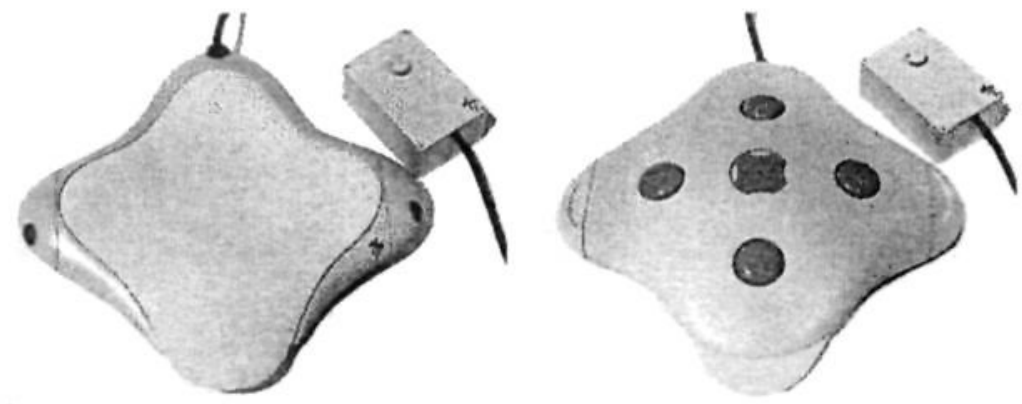

Figure 3 Mojo - interactive seat for VE navigation 


\section{CONCLUSION}

The research by Neale et al [6] and Cobb [1] showed that there are usability difficulties with the joystick and mouse, which were found to be the most suitable devices for people with learning difficulties to control VE tasks. However, this research was not specific about the kinds of difficulties that are experienced with these devices. Therefore it was decided that the next step would be to conduct a thorough evaluation of the joystick and mouse, in order to identify the specific usability difficulties experienced and to clarify how research should progress.

\section{Input device evaluation}

\section{Aim}

To identify the usability difficulties, which young people with learning difficulties experience when using the joystick and mouse for VE navigation and interaction tasks respectively

\section{User Group}

14 students were selected from the Shepherd School in Nottingham to form the user group. The user group attributes were as follows:

- Gender - 7 female and 7 male

- $\quad$ Age range - 7 to 19

- Cognitive ability - 2 moderate/severe and 12 severe learning difficulties

- Physical ability - co-ordination, gross-motor and fine-motor difficulties

\section{Environment}

The evaluations took place at the Shepherd School, in their 'Cyber Café' room. For some evaluations, the room was quiet and mostly free from distraction. During the majority of evaluations, other students would come and go from the room, but would not attempt to disturb the user.

\section{Equipment}

The VEs were displayed on a colour computer monitor and a standard 2-button mouse was used for interaction tasks. 10 students used the Axys joystick (Suncom Technologies) and 4 students used the Wingman joystick (Logitech) for navigation tasks. The stick on the Wingman joystick is much taller and wider than the Axys joystick and is shaped to fit the hand, see figure 4.
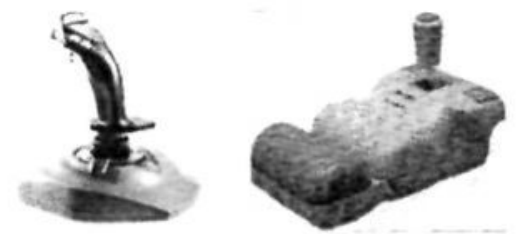

Figure 4 The Wingman joystick (Logitech) \& the Axys joystick (Suncom Technologies)

\section{Task}

Each student was asked to complete navigation and interaction tasks, using the joystick and mouse respectively, within a virtual factory, café or supermarket. Demonstrations of the devices and tasks were given before commencing the evaluations. 


\section{Assessment Measures}

- Misuse of device: non-task related movement, harshness, pressing the wrong buttons, etc.

- Support required: spoken instruction, physical assistance, etc.

- Physical ability: sufficient strength, able to grip properly, etc.

- Workplace: able to reach, etc.

- Attention: on task, on device, on other

- User comments/reactions: positive, negative

\section{RESULTS: USABILITY DIFFICULTIES}

\section{Due to physical ability and device construction}

Approximately half of the students in the user group were able to control the mouse and the joystick they evaluated quite well. However, this group still experienced usability problems with the devices: too much left/right rotation, button misuse, base held still by examiner and grip difficulties with the joysticks; base held still to press button and grip difficulties with the mouse. These difficulties are largely due to the construction of the devices and the physical abilities of the user group, rather than to the user's understanding of how to use each device. It was observed that the Wingman joystick is easier to grip than the Axys joystick, due to its size and shape, highlighting the importance of ergonomic design for increasing usability.

\section{Due to cognitive ability}

The difficulties experienced by other members of the user group are related to their cognitive understanding of how to use the devices: random movement and trying to use for interaction with the joysticks; random movement and frequent pressing of buttons with the mouse. To overcome these difficulties it may be necessary to gain a deeper knowledge of the users' cognitive and perceptual abilities, so that the devices can be refined to an appropriate level of understanding.

\section{Due to task and environment}

As the joysticks and mouse were not specifically designed to be used by people with leaming difficulties to control VEs, there may be certain VE tasks for which they are more difficult to use. This is evident from this evaluation, as physical help was required with all of the devices to complete some tasks by many of the students. Finally, some members of the user group were distracted by other students and activities in the evaluation room. Design guidelines were suggested, which should help to focus the students' attention on the VE, for example, workstation helps to engage the user.

\section{CONCLUSION}

This evaluation has highlighted the importance of considering the physical and cognitive abilities of the user group, as well as the tasks that the user must complete with the input devices, and the environment in which the tasks will be performed, in order to develop a usable computer interface device. This theory is backed up by contemporary human-computer interaction ( $\mathrm{HCI}$ ) research, which also stresses that you should design for the user, the task and the environment [9]. In user-centred design, the first research activity performed is to 'understand and specify the context of use'. This can be achieved by conducting a Usability Context Analysis (UCA), which involves a user, task and environmental analysis. Therefore, it was decided that a user-centred design methodology would be employed in order to ascertain the requirements for the selection or design of usable VE input devices, for young people with moderate/severe learning difficulties. 


\section{User-centred design}

\section{METHODOLOGY}

Usability is a crucial factor in the production of a successful human-computer interface and is central to the user-centred design process. The usability of a product is defined in ISO 9241, part 11 (the British Standard giving guidance on usability) as 'the extent to which a product can be used by specified users to achieve specified goals with effectiveness, efficiency and satisfaction in a specified context of use'. According to the ISO 13407 standard (human centred design processes for interactive systems) the key activities in user-centred design are:

- Understand and specify the context of use

- Specify the user and organisational requirements

- Produce designs and prototypes

- Carry out a user-based assessment

The user-centred design methodology, which has been employed for this research project, was formed by combining established guidelines on user-centred design [10,11) with contemporary human-computer interaction and product design research:

1. Understand and specify the context of use

- Usability Context Analysis (UCA) - user, task and environment analysis

2. Specify the user and organisational requirements

- Identify design requirements (from UCA data)

- $\quad$ Product Analysis (identify device attributes)

- Design Specification (DS)

3. Technology Review

- Virtual reality, assistive and general computer interface devices

4. Produce concept designs and prototypes

5. Carry out a user-based assessment

- $\quad$ Produce evaluation plan (including a Usability Specification)

- $\quad$ Conduct Usability Evaluation

- User-derived feedback used to refine the prototype(s)

Steps $1,2,4$ and 5 are repeated until the usability metrics, outlined in the Usability Specification have been attained, see figure 5 .

\section{User Group and Usability Team}

A user group of 21 students was selected from pupils who attend the Shepherd School in Nottingham. 14 of these students had previously participated in the Input Device Evaluation. Students were selected who seemed to enjoy using computers and showed an understanding of how to use the VEs. The students ranged from age 7 to 19 , with 5 primary, 8 secondary and 8 from the $16+$ department of the school ( 9 of the students were female and 12 were male).

A Usability Team was formed to advise and monitor the project development, by reviewing the UCA data, Design Specification, concept designs, Usability Specification and Usability Evaluation feedback. This multi-disciplinary team included: project advisors; the design engineer; human-computer interaction and special needs experts; a usability specialist and an occupational therapist. 


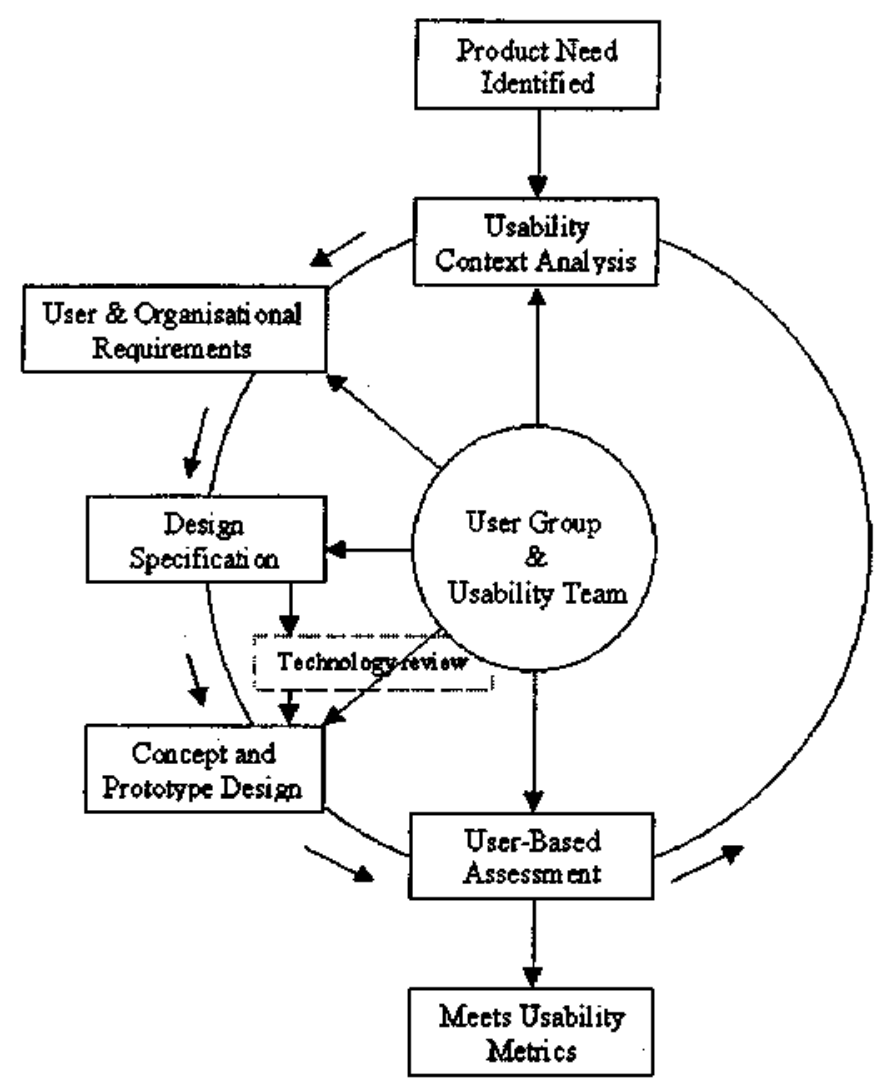

Figure 5 The user-centred design cycle

\section{Understand and specify the context of use}

This activity was achieved by conducting a Usability Context Analysis (UCA), which included a user, task and environment analysis. The UCA guidelines available from Serco Usability Services [12] and the relevant sections of the USERfit toolkit [11] were utilised for this research.

\section{USER ANALYSIS}

Information was gathered about each member of the user group, on their attributes, which could affect the usability of the input device: skills and knowledge; physical, cognitive and perceptual abilities; communication; behaviour and motivations. This data was obtained through various sources, including general observation, the students' educational files and normative assessment tests.

\section{Cognitive ability}

In order to gain some understanding of the cognitive abilities of the user group, two normative assessment tests were used:

- The BPVS-II (British Picture Vocabulary Scale): a test of receptive English vocabulary, which correlates highly with verbal intelligence

- The MAT-SF (Matrices Analogies Test - Short Form): a test of non-verbal reasoning 
The results showed that the students in the user group are generally in the moderate to severe level of cognitive functioning.

\section{Physical ability}

Details of the fine and gross motor abilities of the user group were obtained through the QNST-II (Quick Neurological Screening Test) and the students' educational files. The tasks on the QNST-II provide an opportanity to observe the students' skill in controlling gross and fine muscle movements and their motor planning and sequencing abilities. Table 1 shows some examples of the student's fine and gross motor difficulties.

Table 1 Examples of physical attributes of the user group

\begin{tabular}{|l|l|}
\hline Fine motor difficulties & Gross motor difficulties \\
\hline Clumsy pen grip: 10 & Uses a special chair: 1 \\
\hline Slight hand tremor: 3 & Cerebral Palsy in legs and right arm: 1 \\
\hline Limited wrist movement: 6 & Unsteady arm movement: 2 \\
\hline Motor planning difficulties: 19 & Co-ordination: 14 \\
\hline
\end{tabular}

\section{Perceptual ability}

Details of the perceptual abilities of the user group were also obtained through the QNST-II, with further input from the students' educational files. The main difficulties experienced were with spatial awareness, ordering and sequencing, mixed laterality and bilateral tasks (those involving use of both hands).

\section{TASK ANALYSIS}

A hierarchical task analysis, described by Dix, A et al [13], was carried out. In this process the primary tasks are broken down into subtasks and consequently the subtasks are further broken down, see figure 6 .

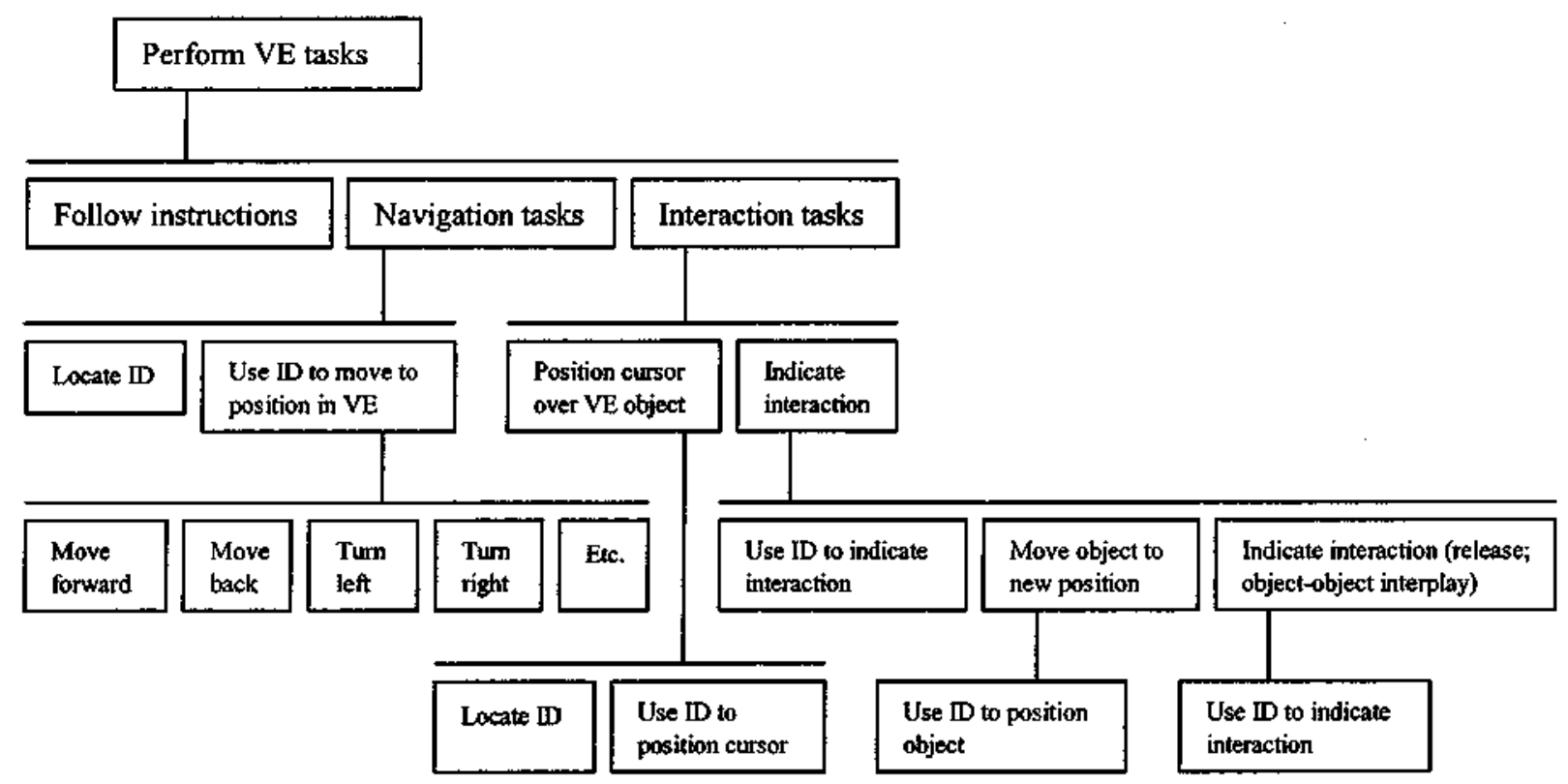

Figure 6 Hierarchical task analysis for VE control 
Environment analysis

This study included an analysis of the organisational, technical and physical factors of the environment in which the product will be used. By gaining an understanding of these factors, a computer input device can be selected or developed to fit the task environment. Table 2 details the organisational factors.

Table 2 Organisational Environment

\begin{tabular}{|l|l|}
\hline Organisation factor & Description \\
\hline Group working & Work alone or in small groups (2 to 3); collaborative VEs \\
\hline Assistance & Teacher/carer may be required \\
\hline Internuptions & Other students may distract user \\
\hline Performance monitoring & $\begin{array}{l}\text { Computer software could record session/level reached; } \\
\text { observation by teacher/carer }\end{array}$ \\
\hline Performance feedback & $\begin{array}{l}\text { Score or time taken given; intelligent agent reports 'well } \\
\text { done', 'better luck next time' }\end{array}$ \\
\hline Pacing & Able to work at own pace or against a clock \\
\hline
\end{tabular}

\section{Specify the user and organisational requirements}

Design requirements were extrapolated from the user, task and environment details obtained through the UCA. These requirements are design decisions, which should help to increase the usability of the computer input device(s) for the user group. A Product Analysis was then conducted in order to describe how the design requirements could be met through specific device attributes. Examples of the design requirements and device attributes are shown in table 3 .

Table 3 Examples of Design Requirements and Device Attributes

\begin{tabular}{|l|l|}
\hline UCA details & Design requirement \\
\hline Age range: 7:05 to 19:00 & Age appropriate appearance (7-19) \\
\hline Slight hand tremor & Will not detect unintentional movements \\
\hline BPVS-II; Extremely low score range & Minimum user input for task completion \\
\hline Spatial awareness difficulties & Provides visual cues to interface and function \\
\hline Design requirement & Device attributes \\
\hline Age appropriate appearance $(7-19)$ & Modern style, attractive colours \\
\hline Will not detect unintentional movements & $\begin{array}{l}\text { Calibration to damp out unintentional } \\
\text { movement }\end{array}$ \\
\hline Minimum user input for task completion & One user action = one VE function \\
\hline Provides visual cues to interface and function & Form indicates interface and function \\
\hline
\end{tabular}

\section{Design Specification}

The device attributes were collated in a design specification and separated into categories, for example, product appearance, ergonomic design, interface and functional assistance, cognitive and physical factors. Additional important aspects to a product's design, which contribute to its success, were added to the design specification. These include: performance, production, life in service and conformance requirements. The design specification is the main input to the concept design stage of the design process and therefore ensures that the design requirements are carried through to the production of a usable prototype. 


\section{Technology review}

The primary aims of the technology review were to identify any existing computer interface devices that, with adaptation, could provide a potential solution and to identify opportunities for innovation. The following areas were reviewed, with reference to the design specification: virtual reality, assistive and general computer interface technology and gaming interface devices. A similar review was conducted before commencing the user-centred design process and hence without the design specification for reference.

\section{FINDINGS}

Several devices were found to possess a few of the device attributes in some of the categories. For ergonomic design, the Anir Ergonomic Mouse (Keytools) has been designed to help avoid repetitive strain injuries (RSI), to partly satisfy 'conforms to health and safety standards'. The FireStorm (ThrustMaster) is a games console, which satisfies 'form indicates interface' and 'haptic sensation with user action', which are interface and function assistance attributes. For physical factors, the Roller Joystick (Penny \& Giles) 'provides assistance to user actions', with its latching drag feature and is of a 'robust construction'.

The Tilting Games Pad was found to be the 'closest concept', see figure 7, matching a significant number of the device attributes listed in the design specification. Visually, it would appeal to the user group, with its 'modern style, attractive colours'. The device is worn by adorning a glove, hence the 'form indicates the interface' (interface and function assistance). The user is required to tilt or wave their hand for the software to react, which suggests that the cognitive factor 'movement in VE same as user action' could be fulfilled. However, the user movements required may not be appropriate for all VE applications.

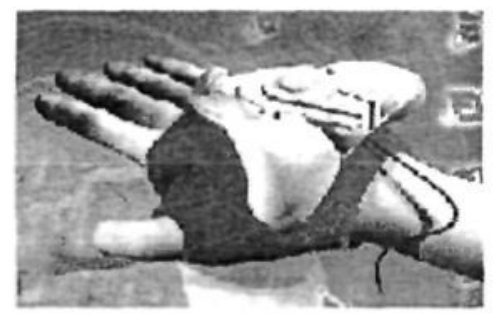

Figure 7 Closest concept - The Tilting Games Pad (Keytools)

\section{CONCLUSION}

As there were no devices identified through the technology review that met all the requirements of the design specification, it can be concluded that there is a need for the design and development of new concepts. Some exciting technology emerged from the virtual reality review, including the PINCH glove system and CAVE C04, see figure 8, which will help to inspire innovation in new concepts. 


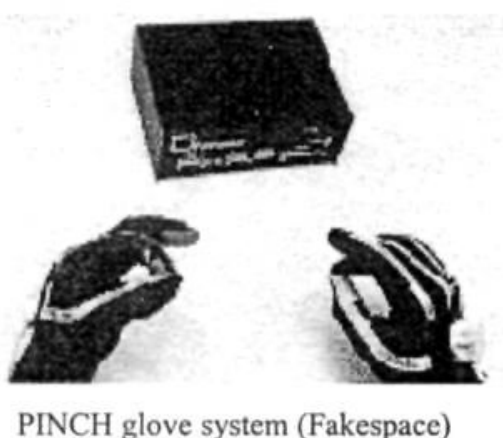

Figure 8 Inspiring technology from the virtual reality review

\section{Future research}

\section{PRODUCE CONCEPT DESIGNS AND PROTOTYPES}

The product design methods described by Baxter [14], for concept and prototype design, will be utilised for this stage of the design process. Initially, many concepts will be generated through employment of the concept generation methods, with reference to the design specification. Concept selection techniques will then be utilised to select the best concept against the design specification. Storyboards and sketches will be used to present concepts to the usability team and user group for modification and new idea generation. The selected concept(s) will go through embodiment design, which takes a concept and develops it to the point at which a full working prototype can be made. Finally, engineering drawings, which are the output from embodiment design, will be followed to produce the evaluation prototype(s).

\section{CARRY OUT A USER-BASED ASSESSMENT}

A user-based assessment of the prototype(s) will be carried out to evaluate the extent to which the user and organisational requirements have been met, and to recommend how the device(s) should be refined. The procedures, which will be followed, are described in the "handbook of user-centred design' [10] and the 'usability engineering' section by Faulkner [9].

An evaluation plan will identify the resources required and the intended methodology, which will describe how the data will be collected. This planning also involves the preparation of a usability specification, which lists the usability attributes and usability metrics. The usability attributes define the success of the prototype(s), i.e. user-satisfaction, and the usability metrics determine how the attributes will be measured. A selected user group of students with moderate/severe learning difficulties will then use the prototype(s) to complete a pre-defined set of tasks within a VE. The results will be analysed and recommendations made to refine the prototype(s).

A process of evaluation, design refinement and re-evaluation will be carried out until the usability metrics, outlined in the usability specification have been attained. The completion of this study should result in the production of a VE interface device for young people with moderate/severe learning difficulties, which satisfies ISO 9241 (the British Standard giving guidance on usability). 


\section{References}

1 Cobb S V G, Neale H R, Reynolds $H$. Evaluation of virtual learning environments, Proc. $2^{\text {tid }}$ European Conf. Disability, Virtual Reality and Assoc. Tech., Skovde, Sweden, 1998: 17-23

2 Standen P. Playing for Real, Mental Health Care,1998, Volume 1, Number 12

3 Donaldson M. Children's Minds, Fontana Press, London, 1978: 19-22

4 Hall J D. Explorations of population expectations and stereotypes with relevance to design, undergraduate thesis, Dept. Manufacturing Engineering, University of Nottingham, 1993

5 Brown D J, Kerr S J, Crosier J. Appropriate input devices for students with learning and motor skills difficulties, N.C.E.T., 1997

6 Neale H R, Brown D J, Cobb S V G, Wilson J R. Structured evaluation of virtual environments for special needs education, Presence: teleoperators and virtual environments, 1999, 8(3): 264-282

7 Lannen T L. Mojo: control of virtual environments for people with physical disabilities, undergraduate thesis, Dept. Industrial Design, Brunel University, 1997

8 Lannen T L, Brown D J. Computer interface design to virtual environments for people with leaming and physical difficulties, Proc. ICCHP 2000 (International Conf. On Computers Helping People with Special Needs), Karlsnuhe, Germany, 2000

9 Faulkner C. The Essence of Human-Computer Interaction, Prentice Hall, 1998

10 Daly-Jones $O$, Bevan N, Thomas C. Handbook of User-Centred Design, Serco Usability Services, Teddington, England, 1999

11 Poulson D, Ashby M, Richardson S. USERfit: A practical handbook on user-centred design for assistive technology, Tide European Commission, 1996

12 Thomas C, Bevan N. Usability Context Analysis: A Practical Guide, Version 4.03, Serco Usability Services, 1996

13 Dix, A et al. Human-Computer Interaction, $2^{\text {nd }}$ Edition, Prentice Hall Europe, 1998

14 Baxter M. Product Design: Practical Methods for the Systematic Development of New Products, Chapman \& Hall, London, 1995 